\title{
Pattern of Diffiulties Faced By the Attendants of Admitted Patients in Tertiary Care Unit Coming From Outside The Karachi City
}

\author{
Dr.Fahmida Khatoon ${ }^{1}$, Dr.Zulfiqar Sheikh Prof. Ibrahim Hassan Alzahrani ${ }^{2}$, \\ Dr.Abd-Elnaser Badawy ${ }^{4}$ \\ Assistent Professor United Medical And Dental College,Pakistan ${ }^{1}$ \\ Associate Professor ,Dow University Health Science Pakistan. ${ }^{2}$ \\ Professor and Chairman of Pathology, College of Medicine, King Abdulaziz University, Jedah ${ }^{3}$ Medical \\ biochemistry department,Faculty of medicine,Mansoura university ${ }^{4}$
}

\begin{abstract}
:
OBJECTIVES:

To identify and assess the problems faced by the attendants of patients admitted in Karachi's tertiary care unit who have come for treatment from outside the city area.

METHODS: It was a computer based study on 502 attendants in with the help of a questionnaire. It was conducted in Jinnah Postgraduate Medical Centre, Karachi.

CONCLUSION: The attendants faced numerous difficulties at every step. While getting their loved ones treated the attendants themselves are very likely to contract some illness because of these problems. The root of all problems is lack of faculties and resources in their own area. Now the challenge ahead lies in moving beyond documenting the pattern of difficulties to addressing them and putting forward long term solutions.
\end{abstract}

\section{Introduction}

Alleviating the difficulties faced by attendants of patients from outside the Karachi city area admitted in tertiary care unit requires an understanding of causes \& consequence of their problems.

They have to face these difficulties mainly due to lack of facilities in their own area, like in India where $73.7 \%$ of all cases received no prenatal care \& went to hospital after crossing the safety margin \& have to travel difficult terrain in unfavorable climate in doing so ${ }^{1,2}$.

This pattern of difficulties faced by attendants jeopardizes their mental \& physical health \& personal hygiene. They make do with whatever food \& drinking water is available which may be unhygienic \& may contain hazardous additives. Asthmatics \& patients of chronic lung or heart disease are particularly vulnerable to outdoor air pollutants to which they are exposed by traveling long distances and inappropriate / lack of shelters. Overcrowding, inability to maintain personal hygiene from lack, unavailability and deplorable sanitation of washrooms ${ }^{3}$, sharing or using sub standard instruments of personal use e.g. razors, combs, etc. greatly increases their risk of developing a serious infection, life threatening disease (e.g. AIDS, Hepatitis-C), nutritional deficiencies \& naturally compromised immune system ${ }^{4}$.

Disturbance is felt not only in their own professional \& social routine but also in their families' back home. This only adds to the mental stress of the attendant, which predisposes them towards psychiatric \& psychological problems 5 .

Financial support by NGOs is given on individual basis to those from outside the Karachi city area seeking treatment in a tertiary care unit. If this finance is spent on development $\&$ true functioning of a three tier health care delivery system, large scale $\&$ long term benefits can be reaped ${ }^{6}$. Also public - private partnerships should be facilitated for this purpose $\mathrm{f}^{7,24}$.

Most important solution is reducing the unnecessary burden of rural attendants on tertiary care units. Then a smaller crowd can be easily managed and provided for. This is achieved in other countries and in Punjab by treating the disease in minor \& sub clinical stage \& preventing them from becoming complicated enough to require the care of a tertiary care unit ${ }^{6}$.

This research primarily addresses the medical sector \& gives a complementary perspective on issues of health care access to vulnerable rural populations. It points towards the challenges faced by \& the support extended to the rural attendants in a tertiary care unit. 


\section{Materials and Methods}

In order to identify the problem areas \& probable causes behind these problems we formulated a questionnaire under. The questionnaire is in English language. It consists of brief section for demographic data 10 questions related to patient attendant relationship, disease of patient $\&$ expenses of the attendant and patient, 20 questions followed by 2-6 options. These 20 questions encompass the reason behind this journey, accommodation \& means of communication, financial help, food and water available to the attendant as well as mental \& physical health \& personal hygiene of the rural attendant. The sample size was 502 attendants.

JPMC was selected as site for our survey because of being Karachi's biggest tertiary care unit \& within easy access of students because of nearness to our medical college. A one-on-one interview based on the questions from the questionnaires was taken from the attendants by the group members. Around 10mins were spent on each interview. Anticipated language barriers were solved because Non-Urdu speaking attendants were almost always accompanied by at least one Urdu speaking person secondly, because of the presence of Sindhi \& Punjabi speaking members in our research group. The questionnaire was of confidential nature and enabled barriers \& problems, especially those of sensitive nature to be explored in depth with an individual.

All data collected in the form of questionnaire was fed and analyzed on SPSS.

\section{III. results}

Questionnaires were filled after interviewing a total of 502 attendants. Generally people did not mind answering our questions. There were a few cases where people either refused to talk or could not understand our point. Our team questioned each and every problem with generosity and politeness.

The following results cane forward after the analysis of the filled questionnaires.

Time:

Most of the interviews were conducted during the peak rush hours of the hospital in the daytime and the remaining in the afternoon.

\begin{tabular}{||l|l|}
\hline AM & PM \\
\hline $\mathbf{3 5 7}$ & $\mathbf{1 4 4}$ \\
\hline
\end{tabular}

Gender

There were more male than female attendants.

\begin{tabular}{|c|c|}
\hline MALE & FEMALE \\
\hline 268 & 233 \\
\hline
\end{tabular}

\section{Mother Tongue}

Most attendants spoke Sindhi. Some also spoke Balochi, Siraki, Pashto, Punjabi and Urdu. (FIG 1)

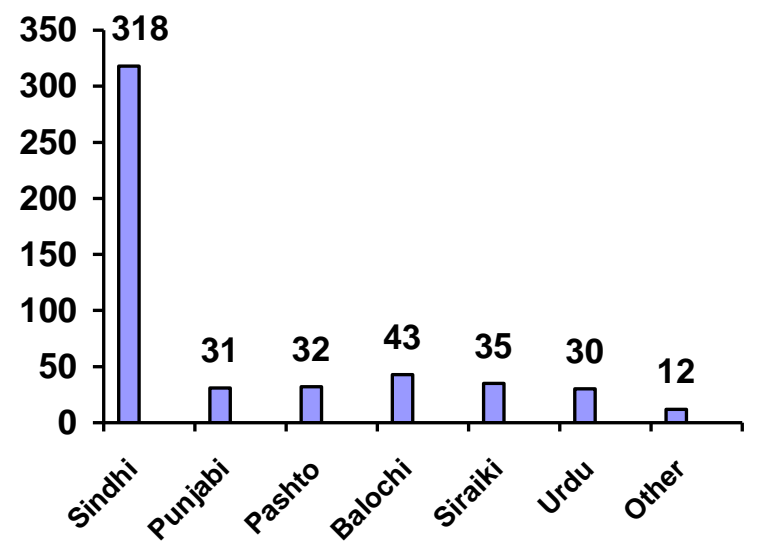

\section{Relationship To Patient:}

\section{Figure 1}

Most of the attendants had parent-child relation ship to patient or were siblings of the patient. Patients were also accompanied by their spouses and cousins. (FIG 2) 


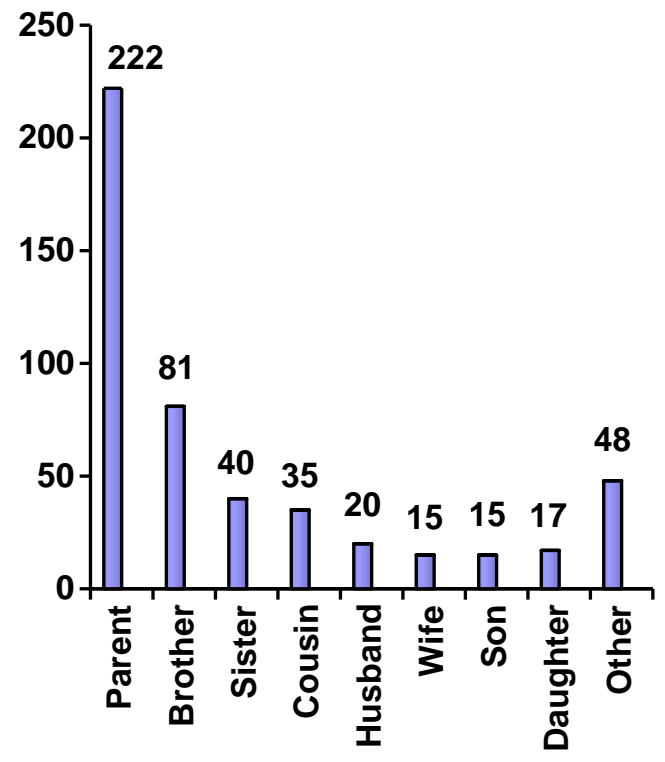

Figure 2

\section{Reason For Coming To Karachi:}

Leaving their home cities and coming to Karachi was basic issue to be addressed. Most common reason cited by the attendants was lack of facilities in their own area and referral by professional doctors. While some followed their personal instincts and advice of family and friends. Still others had miscellaneous reasons. (FIG 3)

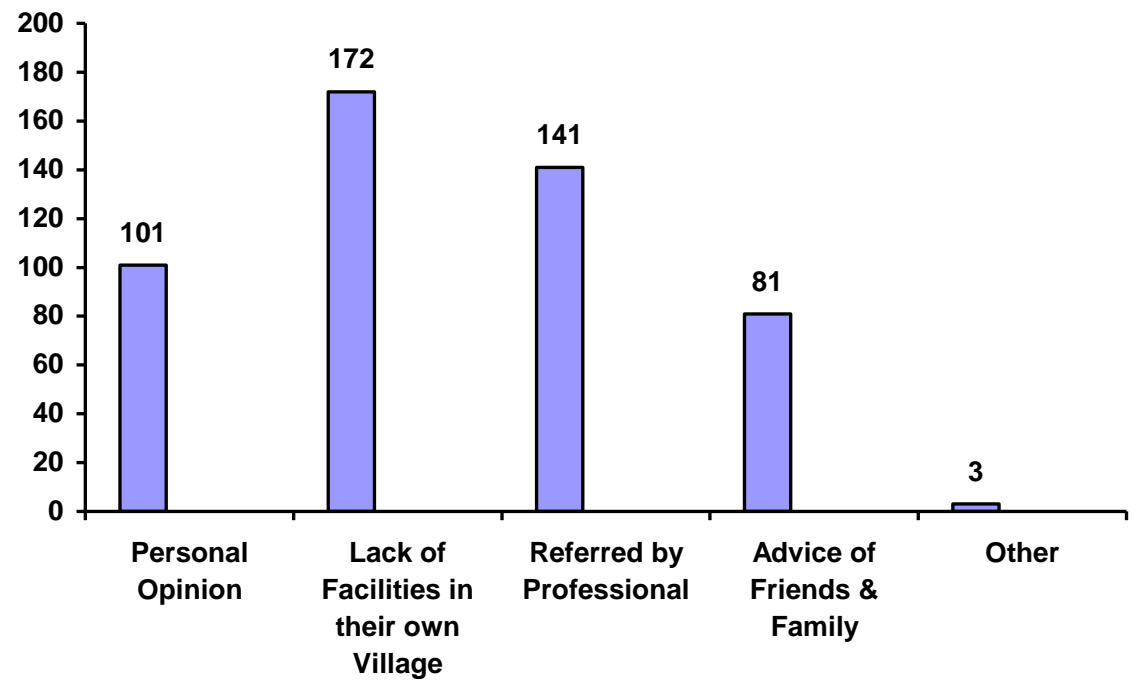

Figure 3

\section{Accomodation \& Shelter Problem:}

A great majority of the people took shelter within the hospital premises. Some had relatives to go to and few could afford staying at hotels. A Small fraction said they had no place to live. Since our research work took place during the peak hot summer so shelter was the major problem being faced by attendants. Almost half of the attendants took shelter within the hospital building, many within hospital premises, some under tree shades, few under shelters and only 5 had no shelter available (FIG 4) 


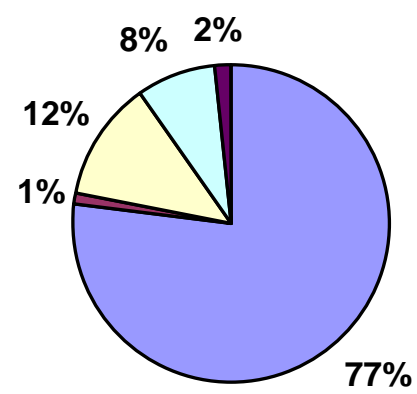

\begin{tabular}{|l|}
$\square$ Hospital \\
$\square$ Hotel \\
$\square$ Relatives \\
$\square$ No Particular \\
Place \\
Other
\end{tabular}

Figure 4

\begin{tabular}{|c|c|c|c|c||}
\hline \hline HOSPITAL & HOTEL & RELATIVES & $\begin{array}{c}\text { NO PARTICULAR } \\
\text { PLACE }\end{array}$ & OTHER \\
\hline 384 & 6 & 60 & 40 & 9 \\
\hline
\end{tabular}

Financial Problems:

Some received help from NGOs and welfare services. No government help was reported.

\begin{tabular}{|l|l|c|}
\hline \multicolumn{1}{|c|}{ YES } & \multicolumn{2}{|c|}{ NO } \\
\hline 106 & 395 \\
\hline
\end{tabular}

\section{Time Allowed With Patient:}

More than half of the attendants were allowed to stay all the time by the patient's side. Some spent an hour or 30 mins or 15 mins with the patient. (FIG 5)

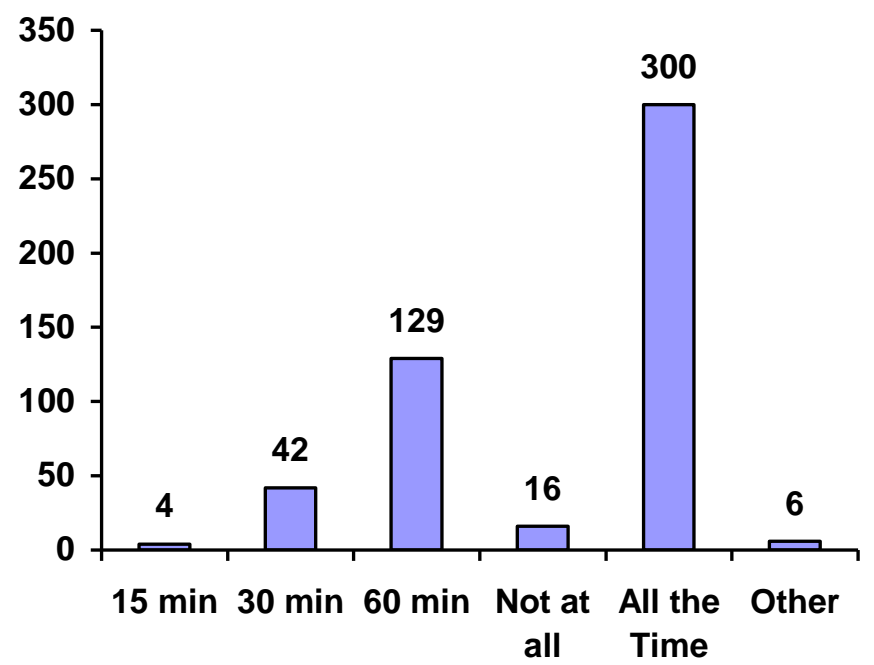

\section{Source Of Food \& Water:}

Figure 5

Food to the attendants was mostly provided by NGOs free of cost. Other common means of obtaining food were hotel followed by cafeteria. No attendant was found to have any means of preparing and cooking their food in the hospital. (FIG 6)

Predictably large number of attendants used tap water for drinking. A small fraction used filtered water and an even smaller fraction used mineral water. While very few had other means. (FIG 7) 

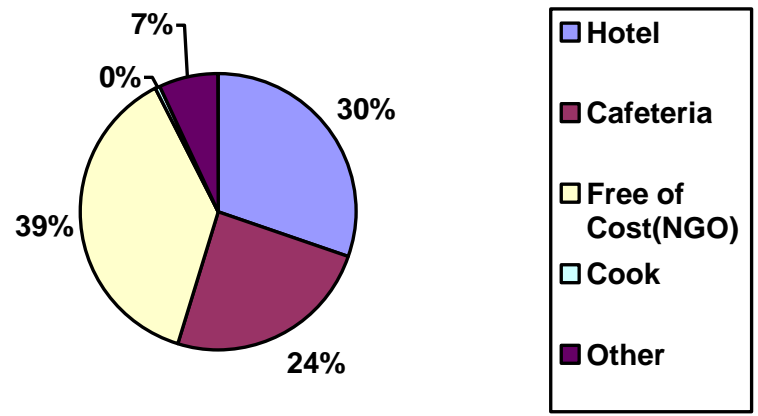

Figure 6 : Food Sources

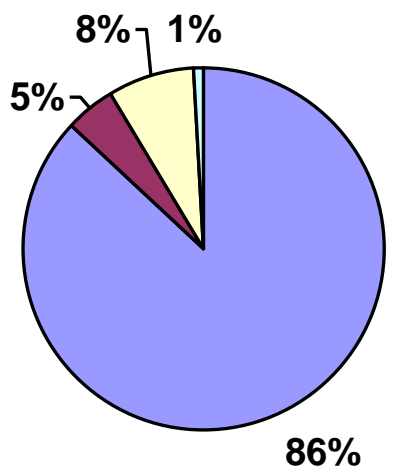

\section{$\square$ Tap \\ $\square$ Mineral \\ $\square$ Filtered \\ $\square$ Other}

\section{Figure 7 : Water Sources}

\section{Availability Of Washrooms:}

Most of the time washrooms were available to most of the attendants but at certain places where male attendants can not enter the wards, availability becomes a problem for some attendants. Few had other means (FIG 8)
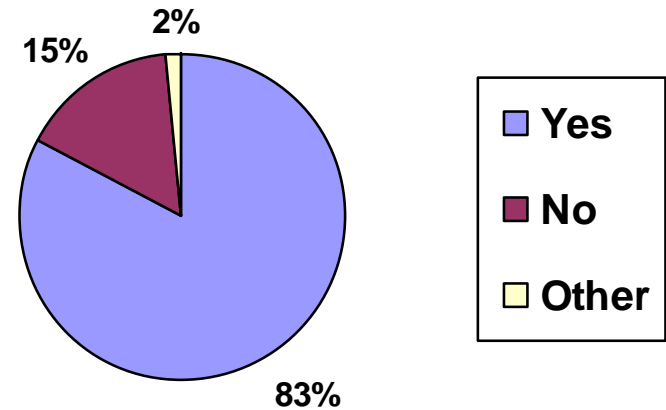

Figure 8

Disturbance Felt By Family Back Home By Their Staying In The Hospital:

Almost all attendants were extremely worried for their families back home even though some had come only after making alternate arrangements for the support of their families in their absence. Very few were not bothered at all with family responsibilities (FIG 9) 

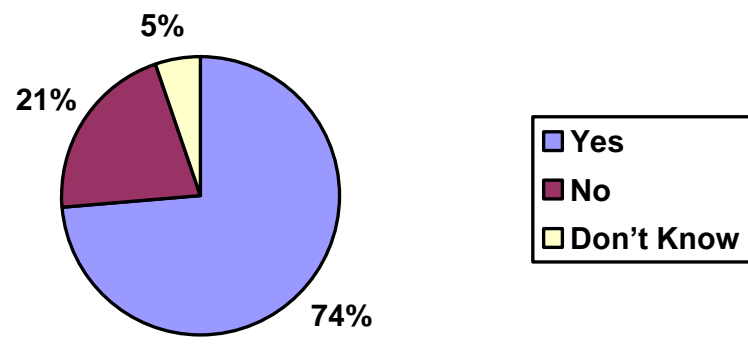

Figure 9

Number Of Additional Attendants:

Approximately half of them were accompanied by 2 or more attendants, many by 1 attendant, and the rest by 3 and more than 3 (FIG 10)

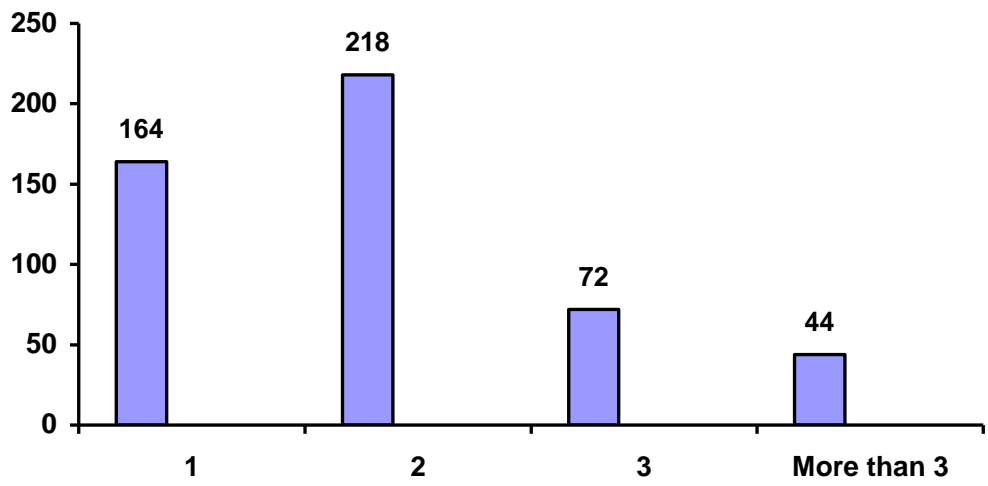

Figure 10

\section{Health Problems:}

Majority of the attendants were satisfied and had not developed any health related problems after coming to the hospital with their ailing loved ones while some complained of common health diseases and others were too busy to realize. (FIG 11)

Most of the attendants claimed to be fit and healthy while some of the female attendants reported weakness. (FIG 12)

Majority of the attendants were not taking any previous medications or suffering from chronic diseases like Hypertension etc. while some did take their routine medications. (FIG 13)

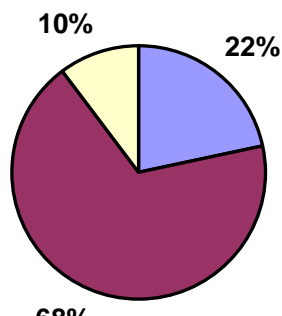

$68 \%$

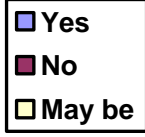

Figure 11 

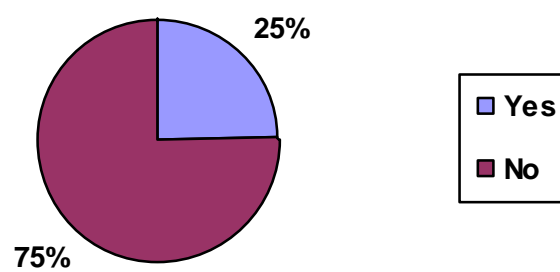

Figure 12
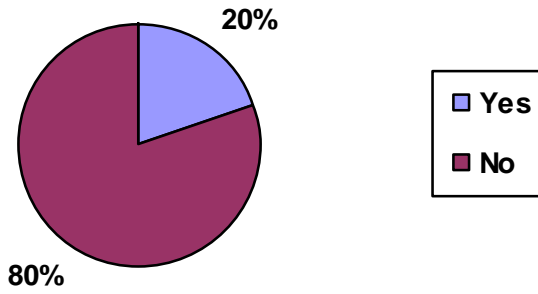

\section{Hygiene Problems:}

Figure 13

Sharing is a major health hazard among attendants (FIG 14)
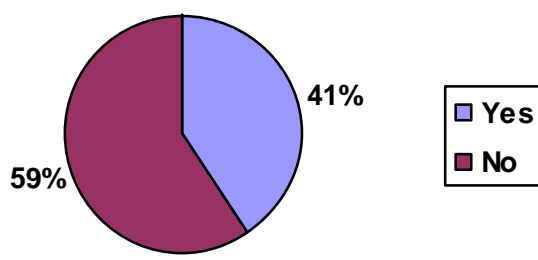

Figure 14

Most of the male attendants were unconcerned about their shaving and looks. While a quarter of interviewed attendants went to roadside barber, some shaved personally and few had other means (FIG 15)

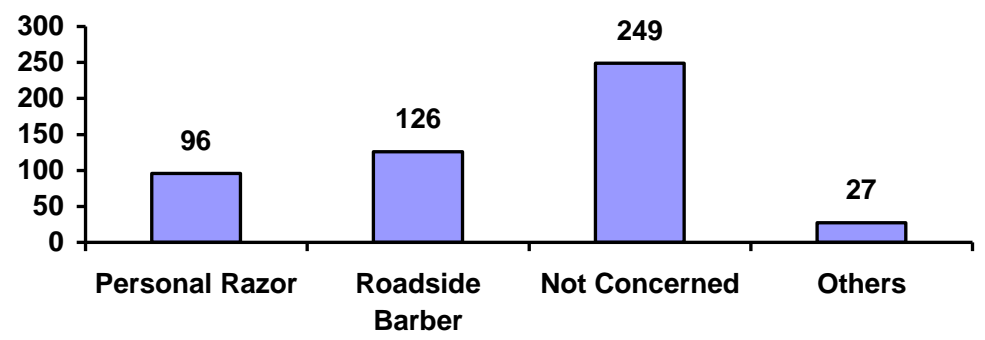

Figure 15

Majority of the attendants claimed to take bath while some did not. (FIG 16) 


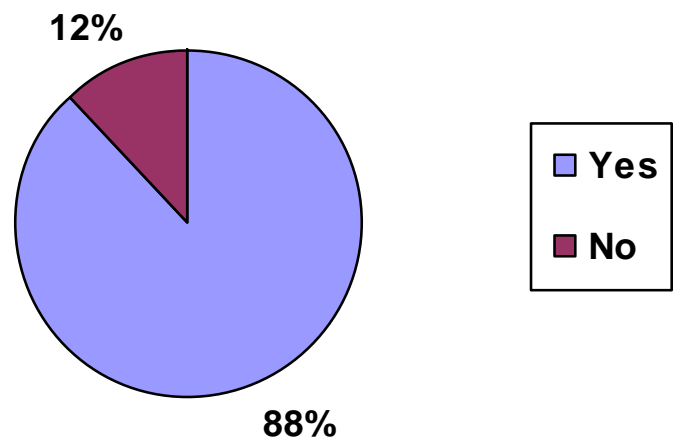

Figure 16

Those attendants, who maintained their cleanliness, took baths mostly after $2-3$ days, some daily, others once a week while few did not bother about it. (FIG 17)

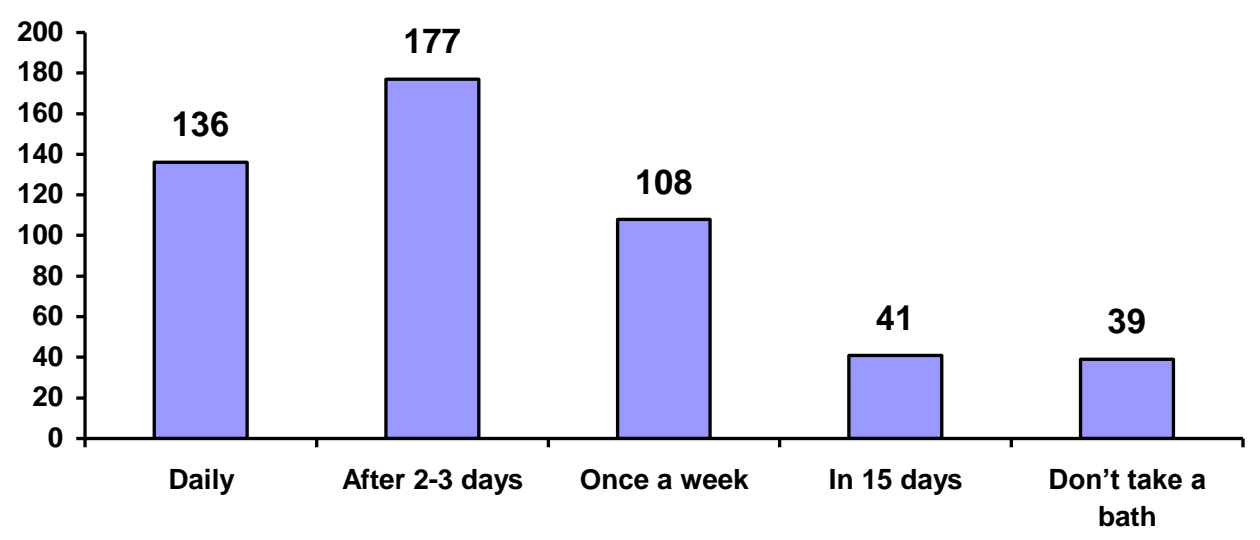

Communication Problems:

Figure 17

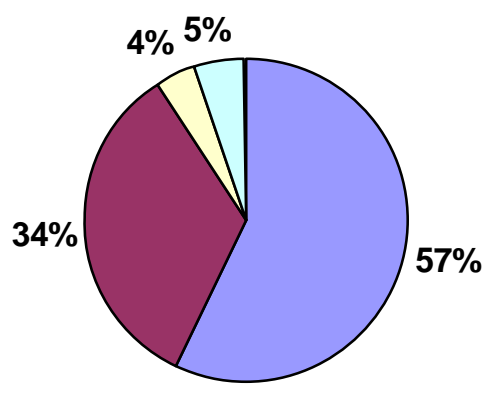

\begin{tabular}{|l|}
\hline$\square$ Mobile \\
$\square$ PCO \\
$\square$ Hospital Services \\
$\square$ No Source \\
\hline
\end{tabular}

Figure 18

\begin{tabular}{|c|c|c|c|}
\hline MOBILE & PCO & HOSPITAL SERVICES & $\begin{array}{c}\text { NO } \\
\text { SOURCE }\end{array}$ \\
\hline 284 & 168 & 20 & 26 \\
\hline
\end{tabular}




\section{Laundry Problems:}

Clothes' washing was another problem. More than half of the attendants washed themselves. Some washed at their relatives' place. Some did not washing them at all. Few took to professional launderers. (FIG 19)

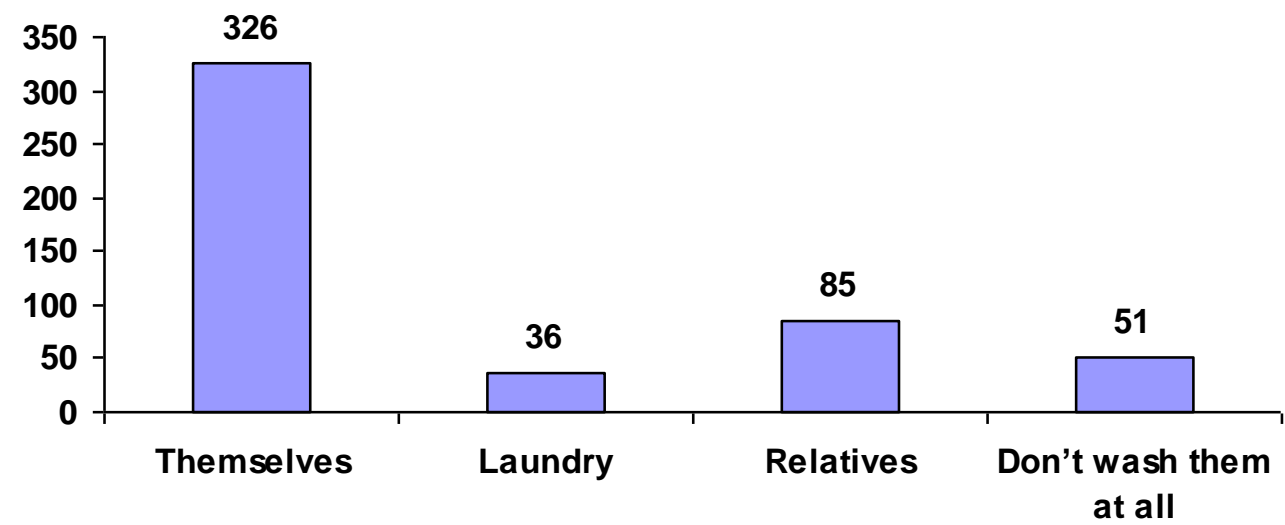

Figure 19

Around half of them washed clothes after 2-3 days, many after a week, some after a month and few did not wash at all. (FIG 20)

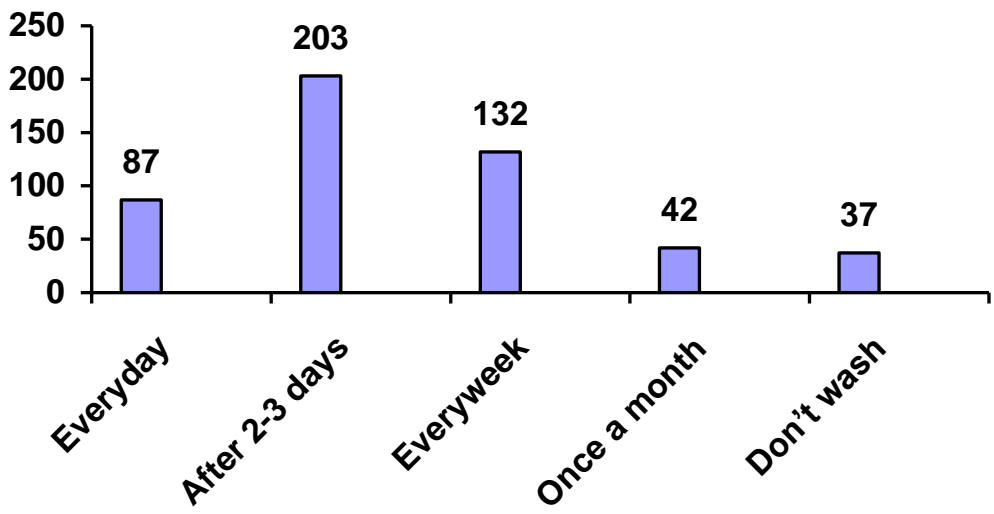

Figure 20

\section{Discussion}

The pattern of difficulties faced by the attendants of admitted patient's is one of the common issues for the citizens, of belonging to low socioeconomic society ${ }^{8}$, which is under a phase of constant negligence. Especially when it comes to government hospitals the attendants face a series of problems since the day of their journey to the hospitals till the admission of patients and afterward.

Lack of facilities in small villages and towns ${ }^{9}$ is the basics cause of botheration and rush in the hospitals of big cities. Most of the rural areas of the country are devoid of basic needs of health and education ${ }^{10}$. Government should take adequate measures to control and plan the basic medical facilities and aids even at the smallest level, as a rite of every citizen of the country, which may be living in urban or rural areas ${ }^{23}$.

Reaching to the city centers for treatment from their villages to major cities gives the attendants and patients a life threatening condition due to underdeveloped and deteriorated conditions of roadways and traffic. Providing more incidences of road accidents and delay in the treatment and thus cost lives of many people ${ }^{11 .}$

During the admission of their patients these poor attendants face a continuous pattern of problems like great expenditure on pharmacy ${ }^{12}$, long waiting for emergency surgeries ${ }^{13}$, rude behavior of medical staff and guards $^{14}$, non-availabities of waiting areas etc. All this adds up anxiety and nervous state of the attendants, who is already suffering from the torture of pain of his close relative's ailment and his half left life at his home town. Situations like this give rise to a feeling of despise and hatred between different, high and low socioeconomic classes as this truly makes them realize their deprivation of basics need of life i.e., health and respect ${ }^{.22}$. 
As most of these people belong to different places of our country, they all can not speak or understand Urdu. Communication problem between the attendants, patients and doctors is another problem. A study based on communication skills is under practice by the Department of Aga Khan University ${ }^{15}$, which is one step towards the resolution but yet, has to be done a lot with.

According to our expectations during the survey, we found no measures, planning or any concern by the authorities about hygiene of food ${ }^{16}$, water, residence, washroom availability and waiting centers for the poor attendant's relief. All these lead to the havoc of hygiene problems. Lack of cleanliness and careless attitude towards hygiene control has not just created problems for the patient's attendants but also for the medical staff ${ }^{18}$, we noticed lack of awareness among the people about contagious diseases like hepatitis $\mathrm{B} \& \mathrm{C}^{19}$ and normal hospital acquired infections. And the attendants are not seen to take no precautions. In sharing their accessories like razors, utensils and usage of contaminated food and water etc. which is source of these health problems.

The hospitals are totally deprived of any sort of prevention from weather extremities that leads to serious problems for the unsheltered attendants. Like, in Karachi, due to monsoon rains, even the busiest hospital life was paralyzed to the extreme as there is no prevention and measures to control the rain and sewage water ${ }^{20}$.

After all this survey of different problems we would specifically like to highlight a study carried at the Hamdard University Hospital and PECHS Trauma and General Hospital about a day surgery program ${ }^{21}$. In this program there is admission of selected patients for planned surgical procedure, patients of certain selected surgeries are returned on the same day, in order to save the botheration of staying admitted in hospital for days, which is a relief not just for the patients but also for their attendants who could return back to their homes immediately.

\section{Conclusion}

We are aware of the problems of hospitals as the issue has been discussed a number of times. But no one ever highlighted the difficulties faced by attendants especially coming from out side the cities. Our survey revealed a complete careless attitude towards this with poor measures for any sort of relief of these poor attendants including financial help, Shelter and safe food and water etc. They face a constant series of helplessness even for months sometimes.

\section{References:}

[1]. Ray A: Difficulties in obstetric practice, Journal of Indian Medical Association, 1996 Apr, 94(4): 131-2,142

[2]. Sanou A, Dembele M, Theobald S, Macq J: Access and adhering to tuberculosis treatment: barriers faced by patients and communities in Burkina Faso, The International Journal of Tuberculosis and Kung Disease 2004 Dec; 8(12):1479-83

[3]. Brocklehurst C: Sanitation is an institutional orphan, Sci-tech World, Dawn/Guardian News Service, 2007 Sept $22: 8$

[4]. Kumar et al: Robbins \& Cotran Pathologic Basis of Disease, 2004, 7/e, Elsevier: chapters 8-9, pgs 343-415

[5]. David Sue et al: Understanding Abnormal Behavior, 2000, 5/e, Hughton Misslin Company: 124-125

[6]. Google, http://www.punjab-prmp.gov.pk/pfis/documents/health.pdf

[7]. Ormond BA, Wallin Sw, Goldenson SM: Supporting the rural Health Care Safety Net, Urban Institute Publications. http://www.urban.org/url.cfm?ID=309437

[8]. Hashim S K, A Socioeconomic and Service Audit of Oncology Unit at The National Institute of Child Health, Karachi, J Pak Med Assoc, May 2003;53(5):205-7

[9]. Sabihuddin Ghausi, Woes of rural Sindh, the DAWN, Monday, November 20, 2006

[10]. Google search, http://64.233.183.104/search?q=cache:bysly_cXKYUJ:www.pakistan.gov.pk/divisions/lgrddivision/media/leftpanel/mtdf/MTDS.pdf+poor+transport+conditions+in+rural+areas+of +pakistan\&hl=en \&ct=clnk\&cd=8\&gl=pk

[11]. Amar Guriro, Baby born in ambulance on Sept 11 never stood a chance, Daily Times, Tuesday, September 18, 2007

[12]. Elizabeth Querna, High drug prices, U.S.News and World Report, Thursday, October 4, 2007

[13]. Masood Jawaid, Muhammad Farhan Amin, Rehan Abbas Khan \& Syed Abdullah Iqbal, An Audit of Reduction in waiting times for emergency surgeries in a tertiary care teaching hospital, Pak J Med Sci October-December 2005 Vol.21 No. 4 422-425

[14]. Farzeen Khalid Hashmi, Shoaib Khalid Hashmi, Attitudes of attendants and visitors at the National Institute Of Child Health (NICH): Problems and Sloutions, Pak J Med Sci January-March 2006 Vol. 22 No. $194-96$

[15]. Waris Qidwai, Syed Irfan Karim, Farhana Masood Irfan, Communication skills of Family Physicians in a Doctor-Patient consultation, J Coll Physicians Surg Pak, Nov 2003;13(11):674

[16]. Hospital hygiene 'risks patients' lives', BBC News, Monday, 21 August, 2000, 07:10 GMT 08:10 UK

[17]. Faiza Ilyas, KARACHI : Poor hygiene at Korangi hospital, DAWN the internet edition, September 08, 2007 Saturday

[18]. Masood Hussain Rao, Knowledge, attitude and practice patterns of handwashing in major public sector hospitals of Karachi, Pakistan Journal Of Medical Research, Dec 2006 45(4): 87-92

[19]. KARACHI: 5,348 hepatitis patients in Sindh, says minister: $170 \mathrm{~m}$ affected worldwide, DAWN the internet edition, Tuesday, June 20, 2006

[20]. Emergency ward submerged again, International The News, August23, 2007

[21]. Mahmood Yousuf, Humaira Jamal Yousuf, Shahid Amin, Mudabbir Hussain, Day Surgery: A need for guidelines, JSP Jul-Sep 2003 Vol 08 No. 03

[22]. Google search, http://www.idsn.org/tekst/national_research.htm

[23]. Iram Noor Muzaffar, The resilient souls of Dhabeji, YOU! ,Tuesday, September 04, 2007

[24]. Ricketts TC: The changing nature of rural health care, Annual Review of Public Health 2000; 21: 639-57 